\title{
Graph-based Kinship Recognition
}

\author{
Yuanhao Guo*†, Hamdi Dibeklioğlu*, Laurens van der Maaten* \\ *Pattern Recognition \& Bioinformatics Group, Delft University of Technology, The Netherlands \\ ${ }^{\dagger}$ Section Imaging \& Bioinformatics, Leiden University, The Netherlands \\ yuanhaoguo2@gmail.com, h.dibeklioglu@tudelft.nl, 1.j.p.vandermaaten@tudelft.nl
}

\begin{abstract}
Image-based kinship recognition is an important problem in the reconstruction and analysis of social networks. Prior studies on image-based kinship recognition have focused solely on pairwise kinship verification, i.e. on the question of whether or not two people are kin. Such approaches fail to exploit the fact that many real-world photographs contain several family members; for instance, the probability of two people being brothers increases when both people are recognized to have the same father. In this work, we propose a graph-based approach that incorporates facial similarities between all family members in a photograph in order to improve the performance of kinship recognition. In addition, we introduce a database of group photographs with kinship annotations.
\end{abstract}

\section{INTRODUCTION}

As a special type of social relationship, kinship plays an important role in the field of social network analysis. Imagebased kinship recognition algorithms try to recognize kinship between people based solely on photographs of their faces. Such kinship recognition may be helpful in uncovering and analyzing social networks, and has applications in surveillance and in criminal investigations. Image-based kinship recognition is a challenging problem: it is a hard task even for humans to recognize kinship among people based on facial similarities. Encouragingly, some recent studies have demonstrated the possibility of kinship verification by means of image-based approaches [1], [2] that identify facial patterns that people may have inherited from their parents. In particular, siblings have the same gene sources which results in the presence of similar facial features. Facial cues that are informative for kinship recognition include the color and shape of the eyes, eyebrows, nose, and mouth [3].

Prior work on image-based kinship recognition has three main limitations. First, prior studies only consider kinship verification: they try to determine whether or not kinship exists between a pair of faces, but they do not aim to recognize the exact type of kinship [2], [4], [5], [6]. Second, current kinship databases are insufficient for the evaluation of existing kinship recognition algorithms, in particular, because existing databases do not contain examples of siblings. Third, prior studies only consider settings in which kinship needs to be verified between pairs of people. This does not correspond to the typical setting encountered on social network websites, on which people often upload photographs that contain more than two family members. One may deal with this problem by separately classifying all pairs of faces in the family picture, but such an approach fails to share information between

\footnotetext{
Most of this work was conducted at Pattern Recognition \& Bioinformatics
} Group, Delft University of Technology. the pairs of people and may produce classifications that are inconsistent (e.g., two people may be classified as sisters whilst they are also classified as having different parents).

Motivated by the aforementioned problems of prior work in kinship recognition, in this paper, we study image-based kinship recognition in photographs that contain several family members. Specifically, the paper makes three main contributions. First, we focus on kinship recognition instead of kinship verification: we aim to recognize the type of kinship relations between people. Second, we introduce two new databases: (1) an annotated database containing photographs of siblings and (2) an annotated database of family photographs. The latter database and part of the former database will be made publicly available (we cannot release all images because of copyright issues). Third, we propose a novel graph-based algorithm that performs joint kinship recognition of all faces in a family picture. The general framework of this algorithm is illustrated in Figure 1. The key advantage of our graphbased algorithm is that it exploits the fact that in a family, the recognized kinship of a particular pair of faces provides evidence for (non)kinship between other pairs of people. For example, in a family, two siblings should have the same father and mother ${ }^{1}$ : if $\mathrm{A}$ and $\mathrm{B}$ are brothers and $\mathrm{C}$ is the father of $\mathrm{A}$, then $\mathrm{C}$ must also be the father of $\mathrm{B}$. Our graph-based algorithm constructs a fully connected graph in which faces are represented by vertices and kinship relations between pairs of faces are represented as edges. Using a few simple kinship rules (that are shown in Table I), we can generate all valid kinship graphs. For each new test image, the predicted kinship graph is the one that obtains the highest score when we sum all scores of the pairwise classifiers that correspond to the edges. Because our graph-based algorithm shares information between the pairwise classifiers, ambiguities in the pairwise kinship classifications may be resolved, which may lead to improved performance. The results of our experiments demonstrate that the proposed algorithm can substantially improve kinship recognition accuracy.

\section{PREVIOUS WORK}

Most prior studies on image-based kinship recognition aim to solve the kinship verification problem using computer vision and machine learning techniques [2], [4], [5], [6]. All these approaches extract facial features and train a kinship verification classifiers on a collection of annotated examples. In the first paper on automatic kinship detection [2], facial resemblance is represented by the difference between facial features. The extracted features include face color, the position and shape

\footnotetext{
${ }^{1}$ In this study, step relationships are not considered.
} 

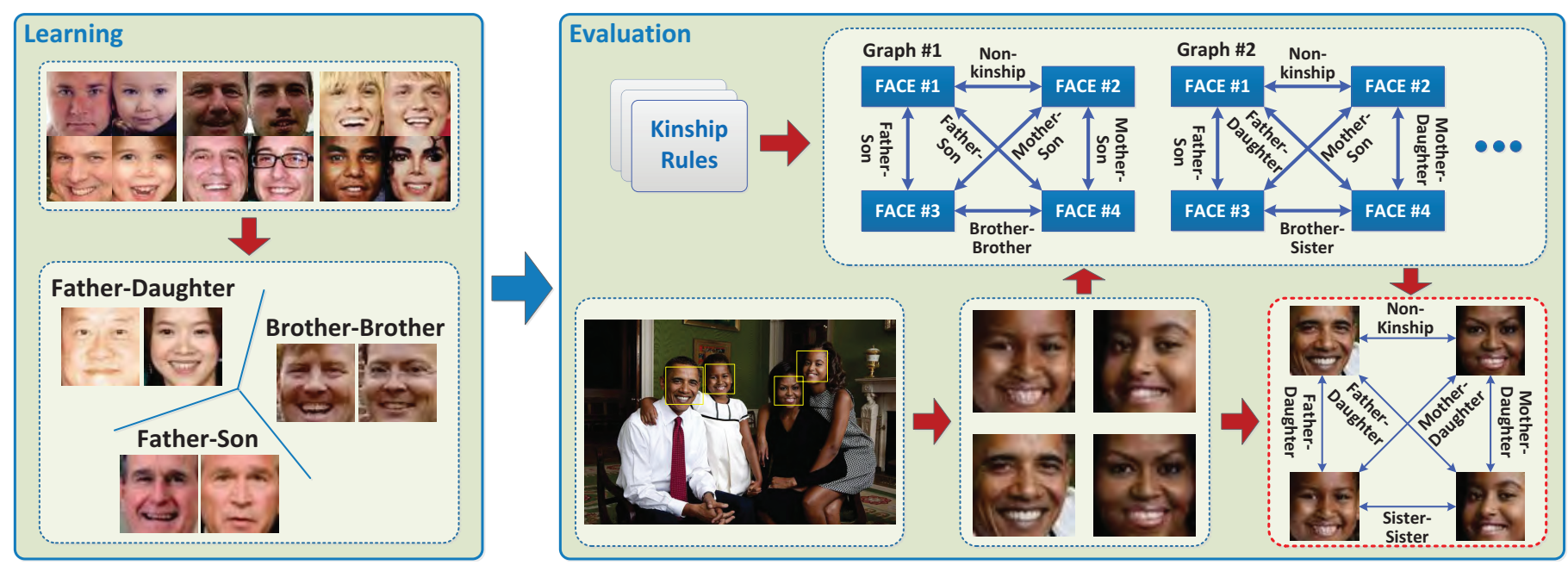

Fig. 1. Overview of the proposed kinship recognition system. In the learning phase, a multi-class kinship classifier is jointly trained on different kinship relations. In the evaluation phase, the faces in family photographs are detected, cropped, and normalized. The set of all valid kinship graphs is generated according to the constraints on kinship relations. For each resulting candidate graph, the classifier scores are summed to obtain an overall score. The kinship graph with the highest overall score is selected as the prediction.

of face parts, as well as gradient histograms. Face parts are localized using a pictorial structures model [7]. Classification is performed using a k-nearest neighbor classifier. [2] presents experiments in which the performance of an automatic kinship verification system is compared with human performance; the results show that the proposed algorithm performs $4.9 \%$ better than human accuracy on this task. [5] improves over this method by dropping the assumption that kinship examples have higher feature similarities than non-kinship examples. They learn a distance metric that aims to repel non-kinship samples as far as possible, whilst kinship samples are pulled close. The method of [5] also combines different types of feature descriptors by learning a multiview distance metric.

In [4] and [8], Xia et al. propose to use transfer subspace learning methods for kinship verification. They exploit the idea that the kinship verification between children and their parents is easier when the parents are young. The method learns a subspace in which old parents and their children are projected close together; the subspace model can then be used to make images of parents look younger. Recently, Dibeklioğlu et al. have proposed a method that uses facial expression dynamics combined with spatio-temporal appearance features to verify kinship in videos [6]. This method is based on the observation that the dynamics of facial expressions are informative for kinship recognition based on videos of people.

In contrast to the aforementioned methods, [9] does not focus on kinship verification but aims at recognizing whether or not a group picture is a family picture. The method estimates the gender and age of every face in the group picture. An image graph is constructed by fitting a minimum spanning tree based on the face locations. Subsequently, the image is represented as a bag of image subgraphs. The resulting bag-ofimage-subgraph features are then used to determine whether or not the group picture is a family picture. The method, however, does not recognize the types of kinship that are present within the family picture.

Our work has several differences in comparison to prior
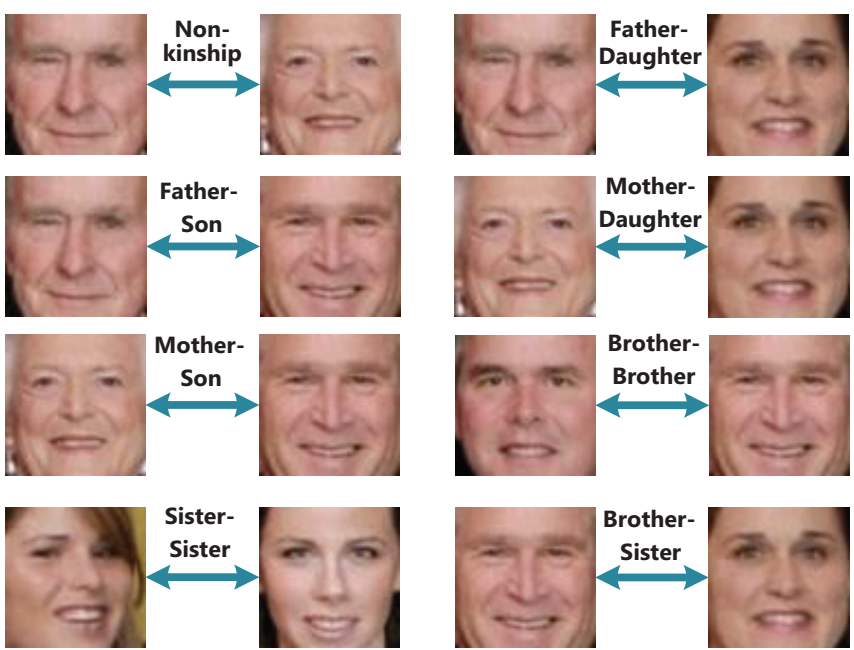

Fig. 2. Normalized face pairs (from the Group-Face database) showing different kinship relations.

studies. First of all, instead of verifying kin relationships, our study focuses on recognizing the exact type of kinship relations. Additionally, our study is the first attempt to generate complete kinship graphs for family photographs.

\section{GRAPH-BASED KINSHIP RECOGNITION}

In this paper, we propose an automatic kinship recognition system that relies on graph-based optimization of multi-class kinship classification. This work does not consider kinship verification between face pairs but focuses on classifying the type of kin relations. Assuming that kin pairs are known in a given group photograph (or predicted by an existing kinship verification system), our system predicts a kinship graph that describes the kinship relations between the family members. 


\section{A. Feature Extraction}

For the reliability of similarity analysis, face images need to be aligned before the feature extraction step. To this end, eye corners are located using the facial landmarking method proposed in [10]. Based on the eye locations, faces are aligned (in terms of roll rotation, translation, and scale) and cropped. Resulting faces images have a resolution of $64 \times 64$ pixels. Figure 2 shows samples of the normalized faces.

To describe the facial appearance, we use local binary pattern (LBP) features [11]. Following [6], LBP features are extracted from each cell in a $7 \times 5$ grid that is laid over the normalized face. In addition to LBP appearance features, we also extract gender and age features from the face images.

In order to estimate a gender feature $f_{\text {gender }}\left(I_{i}\right) \in\{-1,+1\}$ for a given face image $I_{i}$, we classify LBP and bio-inspired features (BIF) [12] using a binary support vector machine (SVM) classifier (with radial basis function kernel). Additionally, we extract an age feature $f_{\text {age }}\left(I_{i}, I_{j}\right) \in\{-1,0,+1\}$ that describes the relative age of the given face images $I_{i}$ and $I_{j}$ :

$$
f_{\text {age }}\left(I_{i}, I_{j}\right)=\left\{\begin{array}{rl}
-1: & a\left(I_{i}\right)<a\left(I_{j}\right) \\
0: & a\left(I_{i}\right) \cong a\left(I_{j}\right) \\
+1: & a\left(I_{i}\right)>a\left(I_{j}\right)
\end{array},\right.
$$

where $a$ denotes the true age of the given subject. For the estimation of $f_{\text {age }}$, we employ a three-class SVM classifier using BIF features. To obtain the final feature vector for a pair of face images $\left(I_{i}, I_{j}\right)$, all features are concatenated:

$$
\mathbf{x}_{i j}=\left[f_{\mathrm{LBP}}\left(I_{i}\right), f_{\mathrm{LBP}}\left(I_{j}\right), f_{\text {gender }}\left(I_{i}\right), f_{\text {gender }}\left(I_{j}\right), f_{\mathrm{age}}\left(I_{i}, I_{j}\right)\right] .
$$

\section{B. Pairwise Kinship Classification}

We model the resulting feature vectors to able distinguish between different kinship types. Moreover, we aim to predict the direction of these relations. For instance, the estimation for the given images will be that $I_{i}$ is the father of son $I_{j}$ (father $\rightarrow$ son), instead of just indicating that $I_{i}$ and $I_{j}$ have father-son relation. To this end, we define 12 types of directional kinship relations such as father $\rightarrow$ daughter, father $\leftarrow$ daughter, father $\rightarrow$ son, father $\leftarrow$ son, mother $\rightarrow$ daughter, $\quad$ mother $\leftarrow$ daughter, mother $\rightarrow$ son, mother $\leftarrow$ son, brother $\rightarrow$ sister, brother $\leftarrow$ sister, brother-brother, and sister-sister. By using these kinship types, more distant kinship relationships such as grandparents-grandchildren, cousins, and uncle/aunt-nephew/niece may also be inferred if the family picture also contains the "intermediate" people.

We use a multi-class linear logistic regressor as the classifier in our system. For a pair of face images, the predicted label $\mathbf{y}^{*}$ is thus given by:

$$
\mathbf{y}^{*}=\underset{\mathbf{y}}{\operatorname{argmax}} \mathbf{y}^{\top}\left(\mathbf{W}^{\top} \mathbf{x}+\mathbf{b}\right),
$$

where $\mathbf{y}$ is a 1 -of- $K$ label vector. $\mathbf{W}$ and $\mathbf{b}$ denote the classifier weights and bias, respectively. To train the multi-class logistic regressor, we define the class-conditional probability:

$$
p(\mathbf{y} \mid \mathbf{x})=\frac{\exp \left(\mathbf{y}^{\top}\left(\mathbf{W}^{\top} \mathbf{x}+\mathbf{b}\right)\right)}{\sum_{\mathbf{y}^{\prime}} \exp \left(\mathbf{y}^{\prime \top}\left(\mathbf{W}^{\top} \mathbf{x}+\mathbf{b}\right)\right)},
$$

and minimize the penalized conditional log-likelihood $\mathcal{L}$ :

$$
\mathcal{L}(\mathbf{W}, \mathbf{b})=\underset{\mathbf{W}}{\operatorname{argmax}}\left(\sum_{\mathbf{x}} \log p(\mathbf{y} \mid \mathbf{x})-\lambda\|\mathbf{W}\|_{2}^{2}\right) .
$$

Herein, the second term is an L2-norm regularizer that is employed to prevent overfitting. The value of the regularization $\lambda$ is set based on the error measured on a small, held-out validation set.

\section{Kinship Graphs}

A simple way to recognize kinship relations in a family photograph is to classify each pair of faces individually. However, this approach does not share information between the pairwise classifications: if the classifier doubts between two kinship types, individual classification cannot exploit the other kinship relations in the photo to resolve this ambiguity. Individual classification may even produce infeasible kinship graphs. For example, it may predict that two people are brothers whilst predicting that they have different parents. The graph-based algorithm we propose aims to resolve these two problems by: (1) generating all feasible kinship graphs and (2) selecting the kinship graph that obtains the highest score.

A kinship graph can be defined as $G=(V, E)$ in which faces correspond to vertices and edges to kinship relations. In other words, each edge $(i, j) \in E$ has an associated label $\mathbf{y}_{i j}$. Two examples of kinship graphs using three faces are shown in Figure 3. Note that the graph shown in Figure 3(b) is infeasible since it violates the constraints on kinship relations that are given in Table I. In the first step, all possible kinship graphs that satisfy these constraints are generated. It is important to note that the candidate graphs can actually be generated offline. The resulting set of candidate kinship graphs are denoted by $\mathscr{G}$. Afterwards, we assign a score to each of the candidate kinship graphs that measures the (log)likelihood of that kinship graph for the observed family picture. Specifically, we define the kinship graph score as the sum of the kinship classifier scores that correspond to each of the edges in the graph:

$$
s(G \mid \mathcal{I})=\sum_{(i, j) \in E} \mathbf{y}_{i j}^{\top}\left(\mathbf{W}^{\top} \mathbf{x}_{i j}+\mathbf{b}\right)
$$

where $\mathcal{I}$ is the family photo, $G=(V, E)$ is the kinship graph that we are scoring, $\mathbf{x}_{i j}$ is the feature vector extracted from the pair of faces associated to edge $(i, j) \in E$, and $\mathbf{y}_{i j}$ is the corresponding kinship label. We perform kinship graph prediction for family photo $\mathcal{I}$ by maximizing the graph score over the set of all candidate kinship graphs:

$$
G^{*}=\underset{G \in \mathscr{G}}{\operatorname{argmax}} s(G \mid \mathcal{I})
$$

where graph $G^{*}$ is the predicted kinship graph.

\section{NEW DATABASES}

To evaluate our approach, we gathered two new kinship recognition databases: (1) a database with image pairs of siblings and (2) a database with family photographs. Both databases (except some copyrighted images in the first database) will be made available to the research community ${ }^{2}$. Both databases are described separately below.

\footnotetext{
${ }^{2}$ http://visionlab.tudelft.nl/content/kinship-recognition
} 


\begin{tabular}{|c|c|}
\hline Definition & Instance \\
\hline \multirow{2}{*}{ - One child can at most have one father and one mother. } & (A-B:Father-Daughter/Son) $\Rightarrow \neg($ C-B:Father-Daughter/Son) \\
\hline & (A-B:Mother-Daughter/Son) $\Rightarrow \neg($ C-B:Mother-Daughter/Son $)$ \\
\hline - Siblings have the same parents. & {$[(\mathrm{A}-\mathrm{B}:$ Father/Mother-Daughter/Son $) \wedge(\mathrm{A}-\mathrm{C}:$ Father/Mother-Daughter/Son $)] \Rightarrow(\mathrm{B}-\mathrm{C}:$ Sister/Brother-Sister/Brother $)$} \\
\hline - Siblings have the same siblings. & {$[(\mathrm{A}-\mathrm{B}:$ Sister/Brother-Sister/Brother $) \wedge(\mathrm{A}-\mathrm{C}:$ Sister/Brother-Sister/Brother $)] \Rightarrow(\mathrm{B}-\mathrm{C}:$ Sister/Brother-Sister/Brother $)$} \\
\hline - There should not be kinship between father and mother. & {$[(\mathrm{A}-\mathrm{B}:$ Father-Daughter/Son $) \wedge(\mathrm{C}-\mathrm{B}:$ Mother-Daughter/Son $)] \Rightarrow$ (A-C:Non-kinship) } \\
\hline
\end{tabular}

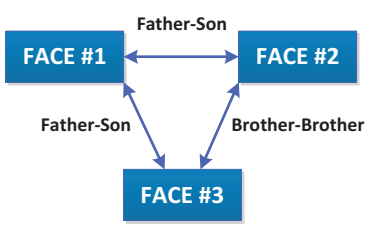

(a)

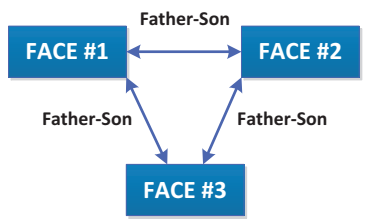

(b)
Fig. 3. Samples of (a) feasible and (b) infeasible kinship graphs.

TABLE II. DISTRIBUTION OF KIN PAIRS (IMAGE PAIRS) IN THE KFW-II, SIBLING-FACE AND GROUP-FACE DATABASES.

\begin{tabular}{lccc}
\hline & KFW-II & Sibling-Face & Group-Face \\
\hline Father-Daughter & 250 & - & 69 \\
Father-Son & 250 & - & 69 \\
Mother-Daughter & 250 & - & 70 \\
Mother-Son & 250 & - & 62 \\
Brother-Brother & - & 232 & 40 \\
Sister-Sister & - & 211 & 32 \\
Brother-Sister & - & 277 & 53 \\
\hline
\end{tabular}

\section{A. Sibling-Face Database}

Existing large-scale kinship databases (such as the KFWII database [5]) do not include sibling pairs. The UvA-NEMO database [13], [6] contains sibling pairs, but it has a small number of subjects. We have gathered a new database that contains more than 200 image pairs for each of three possible sibling relations (brother-brother, sister-sister, and brother-sister). All sibling images have been collected from websites such as Flickr; the sibling relations have been determined based on the tags or descriptions of the images. The sibling faces have been processed in the same way as done for the images in the KFW-II database: they are aligned according to the position of eyes, and resized to a fixed size of $64 \times 64$ pixels. In our experiments, the Sibling-Face database is combined with the KFW-II database to train kinship classifiers. The distribution of kin pairs in the KFW-II and Sibling-Face databases is given in Table II.

\section{B. Group-Face Database}

We have also gathered a collection of group photographs from publicly available sources such as Flickr. Specifically, we have selected group pictures in which the people are all frontally facing the camera. Some samples from the collected database are shown in Figure 4. The database consists of 106 group photographs, of which 82 contain group(s) of family
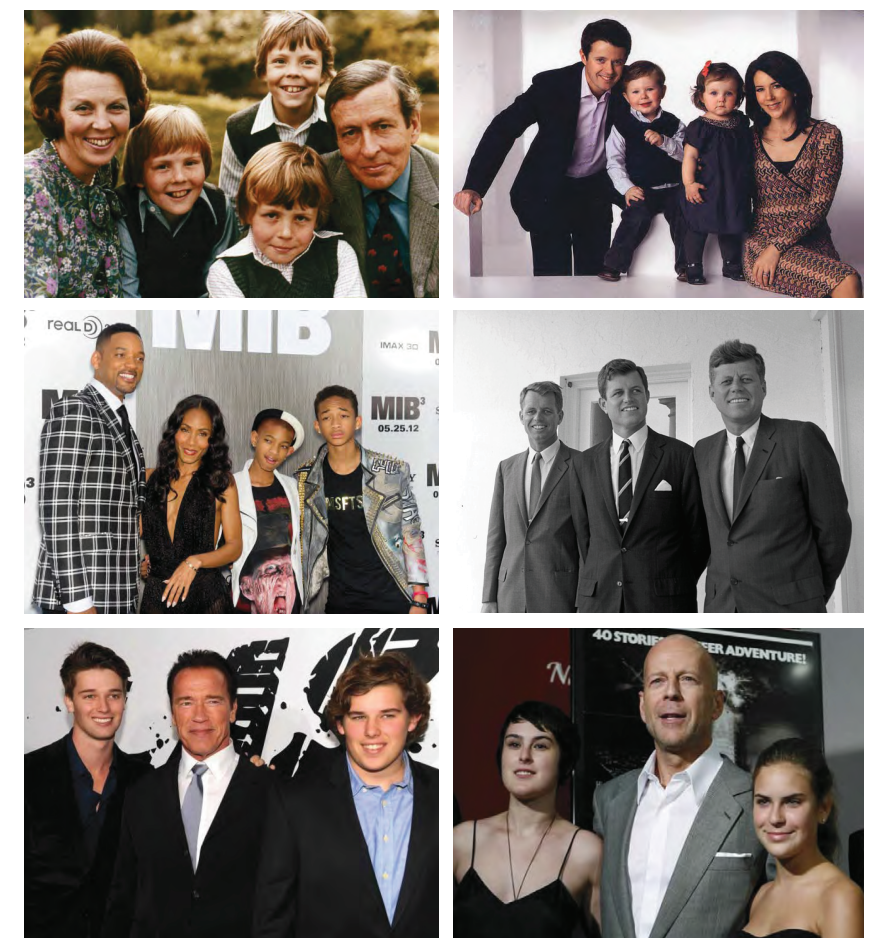

Fig. 4. Sample images from the Group-Face database.

members. To facilitate labeling of the kinship relations, we have selected photographs of famous families (royalty, presidents, Hollywood stars, etc.) and photographs of regular families with reliable kin labels. The Group-Face database contains father-daughter (FD), father-son (FS), mother-daughter (MD), mother-son (MS), brother-brother (BB), sister-sister (SS) and brother-sister (BS) pairs. Table II shows the number of image pairs in each kinship class. All the faces in the database have been cropped and aligned in the same way as the faces in the Sibling-Face database.

\section{EXPERIMENTAL RESULTS}

In our experiments, the KFW-II and Sibling-Face databases are combined and used for training. We employ the family photos in our Group-Face database as the test set. It is assumed that we know which pairs of faces in the family pictures have kinship and which pairs of faces do not, i.e. we assume that we have access to a perfect kinship verification algorithm and focus solely on recognizing what type of kinship exists between two people. In our experiments, the maximum number of family members is limited to four because, in our 
TABLE III. KINSHIP RECOGNITION ACCURACY OF THE PAIRWISE (BASELINE) AND THE GRAPH-BASED APPROACHES.

\begin{tabular}{lccc}
\hline Relationship & Pairwise (\%) & Graph-based (\%) & \# Test Pairs \\
\hline Father-Daughter & 66.15 & 67.69 & 65 \\
Father-Son & 51.72 & 65.52 & 58 \\
Mother-Daughter & 57.81 & 71.88 & 64 \\
Mother-Son & 48.15 & 72.22 & 54 \\
Brother-Brother & 43.33 & 63.33 & 30 \\
Sister-Sister & 34.62 & 76.92 & 26 \\
Brother-Sister & 44.00 & 68.00 & 25 \\
\hline All & 52.48 & 69.25 & 322 \\
\hline
\end{tabular}

current (naive) implementation, the total number of candidate kinship graphs and the required amount of memory drastically increases when more than four faces are used. Specifically, when a family photo contains two parents and four children, we manually split the family into two groups which both have parents and two children. In this way, we obtained 98 kinship groups (16 groups with two faces, 40 groups with three faces, and 42 groups with four faces) that we use in our kinship recognition experiments. The test set we used in our experiments will be made publicly available ${ }^{2}$ (as part of the Group-Face database).

As a baseline approach, we individually perform pairwise classification on each edge of the kinship graph to determine the type of kinship. We set the regularization parameter $\lambda$ (see Equation 4) of the kinship classifier by cross-validating over a small held-out validation set.

To test the reliability and efficacy of the proposed graphbased kinship recognition, we perform two different experiments. In the first experiment, kinship recognition performances of the graph-based and pairwise approaches are compared. In the second experiment, we investigate the effect of age/gender estimation accuracy on the robustness of the graphbased and pairwise methods. To this end, we systematically perturb the gender and age features which are extracted from the test data. The details and results of these experiments are given below.

\section{A. Graph-based versus Pairwise Classification}

In this experiment, the correct classification rates of the graph-based and pairwise approaches are compared. As shown in Table III, the graph-based method proposed in this paper outperforms the pairwise kinship classification by $16.77 \%$ (absolute) on average. This result demonstrates the efficacy of the graph-based kinship recognition. The highest performance of the graph-based method is achieved for the sister-sister relationship with an accuracy of $76.92 \%$.

For further exploration of the results, the confusion matrices for both methods are given in Figure 5. The results suggest that, unlike the pairwise classification, the graph-based approach is able to recover from errors in the age/gender estimations. For instance, the baseline approach often confuses the father-son relation with the brother-brother relation, presumably due to errors in the relative age estimation ${ }^{3}$. By contrast, the graph-based approach corrects most of such misclassifications by incorporating other relations in the graph, and by ensuring that the predicted kinship graph is feasible. This is confirmed by the number of kinship graphs which are correctly predicted (completely) on the Group-Face database. Whilst the graph-based approach correctly predicts 56 of 98 kinship graphs, only 29 kinship graphs are correctly recognized by the baseline method.

\section{B. Effect of Age and Gender Estimation Accuracy}

The results presented in the previous subsection illustrate the potential merits of our graph-based algorithm, which mainly stem from its ability to correct errors in the age and gender estimations. We further investigate the effect of age and gender estimation accuracy in our method. To this end, we randomly generate labels for the relative age classes and gender by systematically changing the error rate. Both the graph-based and pairwise methods are tested using these labels. Figure 6 shows the kinship recognition accuracy as a function of the error level in age and gender estimation. As shown in Figure 6, both methods achieve $100 \%$ classification accuracy when the age and gender ground truths are used: age and gender completely determine the type of kinship relation between two people, if we assume that the given pair has kinship.

The results show that both pairwise and graph-based approaches perform worse when the perturbation rate is increased for gender and age. However, our graph-based method is more robust to gender and age estimation errors than to the pairwise approach. In particular, the graph-based algorithm is less sensitive to incorrect age prediction. This is beneficial because age estimation is a difficult task in real-life conditions, in particular, because age estimates are strongly influenced by changes in resolution, illumination, gender [14], and facial expression [15]. Our graph-based algorithm is more robust to the resulting errors in the age estimates. As shown in Figure 6 (see top right side of the accuracy maps), graph-based approach performs much better than the pairwise classifier in such conditions.

\section{CONCLUSIONS}

In this paper, we have proposed a novel graph-based method to recognize kinship relations in family photos. Our approach models the kin relationships using a fully connected graph in which faces are represented by vertices and edges represent kinship relations. The overall score of each feasible kinship graph is computed by summing classifier scores over the edges of the graph. The graph with the highest overall score is selected as the prediction. The results of our experiments demonstrate that our graph-based outperforms the pairwise kinship classification approach. Moreover, the proposed method guarantees consistency of the predicted kinship graphs.

\footnotetext{
${ }^{3}$ The correct classification rate of the gender classifier, used in our experiments, is approximately $90 \%$ based on 10 -fold cross-validation. Combination of the KFW-II, Sibling-Face, and UvA-NEMO databases is used for the evaluation. 10-fold cross-validation accuracy of the relative age estimator is approximately $65 \%$ on the combination of KFW-II and Sibling-Face databases. Higher error rate in age estimation is mostly due to small size (low resolution) of the face images, which makes facial wrinkles nearly invisible.
} 


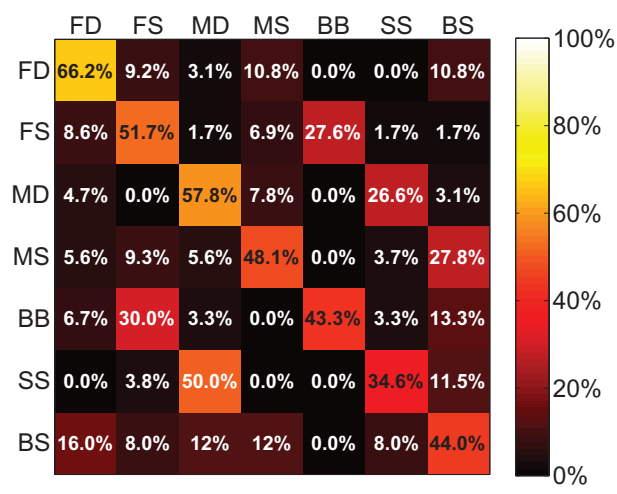

Pairwise Kinship Recognition

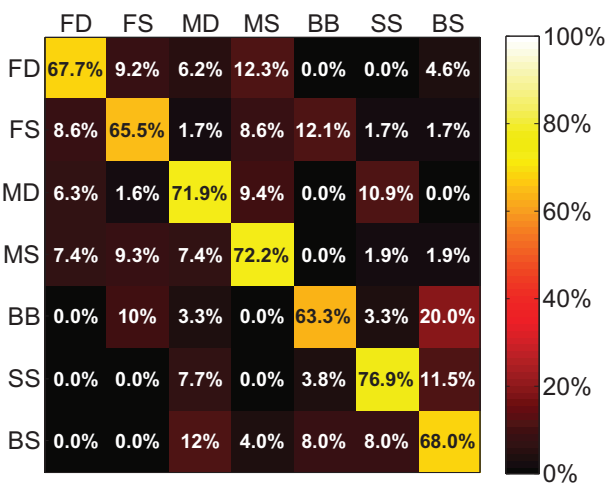

Graph-based Kinship Recognition

Fig. 5. Confusion matrices for the pairwise and graph-based approaches.

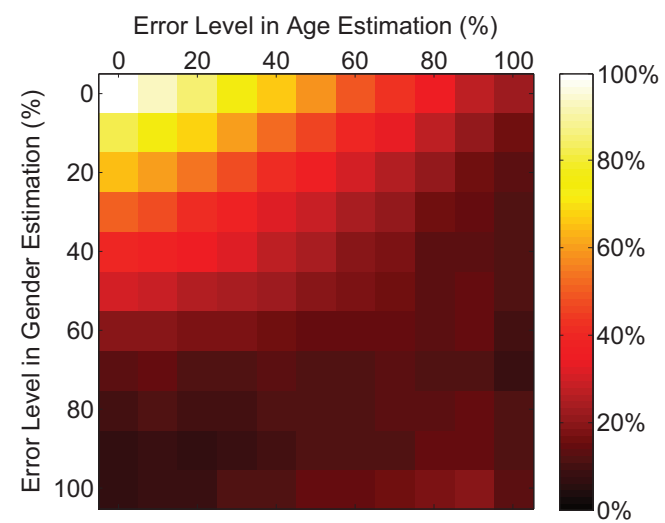

Pairwise Kinship Recognition

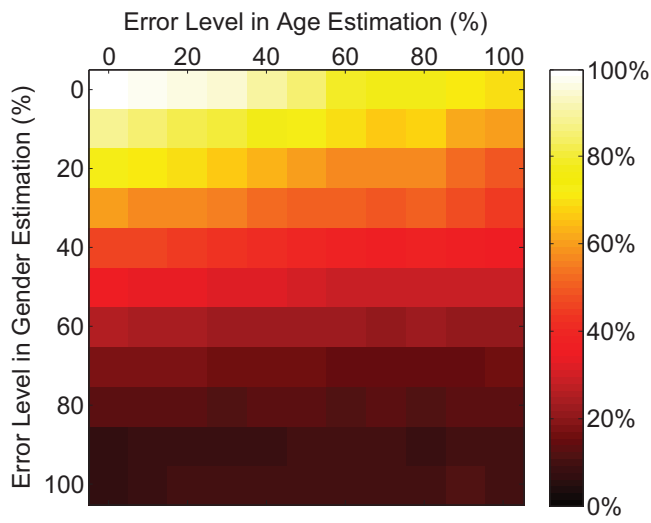

Graph-based Kinship Recognition

Fig. 6. Kinship recognition accuracy (\%) as a function of the error level in age and gender estimation.

As a future direction, we aim to develop a graph-based method to train our kinship classifier as well by framing the task as a structured prediction problem. Also, we aim to improve the speed of our current (naive) implementation by exploiting redundancies in the score computations (like in dynamic programming). Moreover, we plan to include a kinship verification step prior to the classification of relations.

\section{ACKNOWLEDGMENT}

This research was supported by the China Scholarship Council

\section{REFERENCES}

[1] L. T. Maloney and M. F. Dal Martello, "Kin recognition and the perceived facial similarity of children," Journal of Vision, vol. 6, no. 10, 2006.

[2] R. Fang, K. D. Tang, N. Snavely, and T. Chen, "Towards computational models of kinship verification," in IEEE International Conference on Image Processing, 2010, pp. 1577-1580.

[3] M. F. Dal Martello and L. T. Maloney, "Where are kin recognition signals in the human face?" Journal of Vision, vol. 6, no. 12, 2006

[4] S. Xia, M. Shao, and Y. Fu, "Kinship verification through transfer learning," in International Joint Conference on Artificial Intelligence, vol. 3, 2011, pp. 2539-2544.

[5] J. Lu, X. Zhou, Y.-P. Tan, Y. Shang, and J. Zhou, "Neighborhood repulsed metric learning for kinship verification," IEEE Trans. on PAMI, vol. 36, no. 2, pp. 331-345, 2014.
[6] H. Dibeklioğlu, A. A. Salah, and T. Gevers, "Like father, like son: Facial expression dynamics for kinship verification," in International Conference on Computer Vision, 2013, pp. 1497-1504.

[7] P. F. Felzenszwalb and D. P. Huttenlocher, "Pictorial structures for object recognition," International Journal of Computer Vision, vol. 61, no. 1 , pp. 55-79, 2005.

[8] S. Xia, M. Shao, J. Luo, and Y. Fu, "Understanding kin relationships in a photo," IEEE Trans. on Multimedia, vol. 14, no. 4, pp. 1046-1056, 2012.

[9] Y.-Y. Chen, W. H. Hsu, and H.-Y. M. Liao, "Discovering informative social subgraphs and predicting pairwise relationships from group photos," in ACM International Conference on Multimedia, 2012, pp. 669-678.

[10] X. Xiong and F. De la Torre, "Supervised descent method and its applications to face alignment," in IEEE Conference on Computer Vision and Pattern Recognition, 2013, pp. 532-539.

[11] T. Ojala, M. Pietikainen, and T. Maenpaa, "Multiresolution gray-scale and rotation invariant texture classification with local binary patterns," IEEE Trans. on PAMI, vol. 24, no. 7, pp. 971-987, 2002.

[12] G. Guo, G. Mu, Y. Fu, and T. S. Huang, "Human age estimation using bio-inspired features," in IEEE Conference on Computer Vision and Pattern Recognition, 2009, pp. 112-119.

[13] H. Dibeklioğlu, A. A. Salah, and T. Gevers, "Are you really smiling at me? Spontaneous versus posed enjoyment smiles," in European Conference on Computer Vision, 2012, pp. 525-538.

[14] G. Guo, C. R. Dyer, Y. Fu, and T. S. Huang, "Is gender recognition affected by age?" in International Conference on Computer Vision Workshops, 2009, pp. 2032-2039.

[15] G. Guo and X. Wang, "A study on human age estimation under facial expression changes," in IEEE Conference on Computer Vision and Pattern Recognition, 2012, pp. 2547-2553. 